\title{
Correlation-Picture Approach to Open-Quantum-System Dynamics
}

\author{
S. Alipour®, ${ }^{1, *}$ A. T. Rezakhani $\odot,{ }^{2, \dagger}$ A. P. Babu $\odot,{ }^{1}$ K. Mølmer, ${ }^{3}$ M. Möttönen $\odot,{ }^{4}$ and T. Ala-Nissila ${ }^{1,5, \$}$ \\ ${ }^{1}$ QTF Center of Excellence, Department of Applied Physics, Aalto University, \\ P.O. Box 11000, FI-00076 Aalto, Espoo, Finland \\ ${ }^{2}$ Department of Physics, Sharif University of Technology, Tehran 14588, Iran \\ ${ }^{3}$ Department of Physics and Astronomy, Aarhus University, \\ Ny Munkegade 120, DK-8000, Aarhus C, Denmark \\ ${ }^{4}$ QCD Labs, QTF Center of Excellence, Department of Applied Physics, Aalto University, \\ P.O. Box 13500, FI-00076 Aalto, Espoo, Finland \\ ${ }^{5}$ Interdisciplinary Centre for Mathematical Modelling and Department of Mathematical Sciences, \\ Loughborough University, Loughborough, Leicestershire LE11 3TU, United Kingdom
}

(Received 11 May 2020; revised 8 July 2020; accepted 1 September 2020; published 3 November 2020)

\begin{abstract}
We introduce a dynamical picture, referred to as correlation picture, which connects a correlated bipartite state to its uncorrelated counterpart. This picture allows us to derive an exact dynamical equation for a general open-system dynamics with system-environment correlations included. This exact dynamics is in the form of a Lindblad-like equation even in the presence of initial system-environment correlations. For explicit calculations, we also develop a weak-correlation expansion that allows us to introduce systematic perturbative approximations. This expansion provides approximate master equations that can feature advantages over existing weak-coupling techniques. As a special case, we derive a Markovian master equation, which is different from existing approaches. We compare our equations with corresponding standard weak-coupling equations using two examples, for which our correlation-picture formalism is more accurate, or at least as accurate as the weak-coupling equations.
\end{abstract}

DOI: 10.1103/PhysRevX.10.041024

Subject Areas: Quantum Physics, Quantum Information

\section{INTRODUCTION}

The recent rise in high-fidelity quantum technological devices has necessitated detailed understanding of open quantum systems and how their environment influences their performance. The theory of open quantum systems has spurred numerous possibilities to harness the power of the environments in various physical tasks [1,2]. Although in this framework quantum correlations play a key role, it has remained unresolved how to explicitly keep track of correlations between a system and its environment in the dynamical equation.

The description of the joint evolution of a quantum system and its environment, or bath, through the unitary dynamics given by the Schrödinger equation is hampered by the large dimensionality of the Hilbert space of the bath. Although there already exist a plethora of approximate

\footnotetext{
*sahar.alipour@aalto.fi

rezakhani@sharif.edu

†tapio.ala-nissila@aalto.fi
}

Published by the American Physical Society under the terms of the Creative Commons Attribution 4.0 International license. Further distribution of this work must maintain attribution to the author(s) and the published article's title, journal citation, and DOI. methods [3-10] for obtaining dynamics of an open quantum system by a closed set of equations, these approaches, in general, do not incorporate correlations between the system and its environment (see, e.g., Refs. [11-14] for attempts to take correlations into account). Furthermore, despite previous attempts to prove the existence of universally valid time-local Lindblad-like master equations for general dynamics [15-19], a microscopic derivation that incorporates correlations, whether initial or instantaneous, within the dynamics has been elusive.

Here, we address these issues by introducing the correlation picture, a new technique through which we relate any correlated state of a composite system in the Schrödinger picture to an uncorrelated description of that system. The dynamical picture allows us to assign a correlation parent operator to the instantaneous system-bath correlation. This correlation parent operator is used as a key element in a microscopic derivation of the dynamical equation of the system.

This introduced framework for studying open quantum dynamics enables us to derive a universal Lindblad-like (ULL) equation that is time local and valid for any quantum dynamics. The ULL form is derived without approximations, and it is also valid when the system is initially correlated with the bath. A Lindblad-like form for general 
dynamical equations has also been noted in recent attempts to generalize quantum mechanics [20-22].

We study the behavior of the ULL equation under chosen approximations and are able to derive conveniently solvable master equations, which, to a satisfactory degree of accuracy, reproduce the exact dynamics in the corresponding parameter regimes. In particular, in the vicinity of time instants where the correlations become negligible, the approximative ULL equation reduces to a Markovian Lindblad-like (MLL) master equation, in which the jump rates are positive. We prove that this equation correctly characterizes the universal quadratic short-time behavior of the system dynamics [23], in contrast to the standard Lindblad equation, which gives a purely linear behavior for short times $[24,25]$. In addition, we demonstrate that our MLL equation, which does not utilize the secular approximation, may in some cases faithfully describe the long-time behavior of the system. This MLL equation thus constitutes a useful framework for studying open-quantum-system dynamics beyond the weak-coupling regime.

We also develop a systematic perturbative weak-correlation expansion, which includes non-Markovian effects. Interestingly, we show that even the lowest order of the constructed ULL equation (ULL2) can outperform corresponding weak-coupling master equations, which shows that giving the principal role to correlations rather than coupling offers a strong alternative to existing weakcoupling techniques.

\section{CORRELATING TRANSFORMATION}

Any given system-bath state at an arbitrary instant of time $\varrho_{\mathrm{SB}}(\tau)$ can be decomposed in terms of an uncorrelated part, given by the tensor product of the instantaneous reduced states of the subsystems $\varrho_{\mathrm{S}}(\tau)=\operatorname{Tr}_{\mathrm{B}}\left[\varrho_{\mathrm{SB}}(\tau)\right]$ and $\varrho_{\mathrm{B}}(\tau)=\operatorname{Tr}_{\mathrm{S}}\left[\varrho_{\mathrm{SB}}(\tau)\right]$, and the remainder $\chi(\tau)$, which carries all correlations within the total state,

$$
\varrho_{\mathrm{SB}}(\tau)=\varrho_{\mathrm{S}}(\tau) \otimes \varrho_{\mathrm{B}}(\tau)+\chi(\tau),
$$

where $\operatorname{Tr}_{S}[\chi]$ and $\operatorname{Tr}_{B}[\chi]$ are null operators on the bath and system Hilbert spaces, respectively. Below, we refer to $\chi(\tau)$ as the correlation operator or simply the correlation. It includes all kinds of correlations, whether classical or quantum mechanical. The latter, in the form of entanglement, discord, or other more complex types, have a rich and resourceful nature in physics, e.g., in energy fluctuations of thermodynamical systems $[26,27]$ and in quantum information tasks [28-30].

To define our correlation picture, we introduce an operation $\mathscr{E}_{\chi}$, which transforms the uncorrelated state $\varrho_{\mathrm{S}}(\tau) \otimes \varrho_{\mathrm{B}}(\tau)$ to the correlated state $\varrho_{\mathrm{SB}}(\tau)$. We refer to the opposite operation relating the correlated state to the uncorrelated one as decorrelating. These interesting operations also appear in the context of quantum statistical physics, where they are dubbed the quantum Boltzmann map and relate to the stosszahlansatz [31]. Decorrelating transformations have already been investigated in the literature [32], and it is known that a universal decorrelating machine would violate linearity of quantum mechanics [33]. Our correlating transformation, indeed, is not universal; i.e., $\mathscr{E}_{\chi}$ depends on the states. To gain insight on how to find $\mathscr{E}_{\chi}$, it is helpful to start with the case of creating a weakly correlated state. We emphasize that this case study will serve merely to set the scene, whereas later we do not assume any weak correlation in our general analysis.

Correlating transformation for a weakly correlated state. - Let us aim to transform an uncorrelated state $\varrho_{\mathrm{SB}}=$ $\varrho_{\mathrm{S}} \otimes \varrho_{\mathrm{B}}$ to a weakly correlated state $\varrho_{\mathrm{SB}}=\varrho_{\mathrm{S}} \otimes \varrho_{\mathrm{B}}+\chi$ with $\|\chi\| \ll 1$ ( $\|\cdot\|$ being the operator norm). A natural way to do so is to apply an entangling gate on the uncorrelated state. Consider, e.g., an entangling gate [34] described by a unitary transformation $U_{\chi}=e^{-i H_{\chi}}$, with $\left\|H_{\chi}\right\| \ll 1$ [35]. In other words, here the transformation $\left(U_{\chi}\right)$ is assumed to be given, and we look for the corresponding generated correlation operator $\chi$. This gate results in a weakly correlated state $U_{\chi} \varrho_{\mathrm{S}} \otimes \varrho_{\mathrm{B}} U_{\chi}^{\dagger} \approx \varrho_{\mathrm{S}} \otimes \varrho_{\mathrm{B}}-i\left[H_{\chi}, \varrho_{\mathrm{S}} \otimes \varrho_{\mathrm{B}}\right]$. Comparing this state with $\varrho_{\mathrm{SB}}=\varrho_{\mathrm{S}} \otimes \varrho_{\mathrm{B}}+\chi$, we observe that up to $O\left(\left\|H_{\chi}\right\|^{2}\right)$, the correlation $\chi$ obeys the following equation:

$$
\chi=-i\left[H_{\chi}, \varrho_{\mathrm{S}} \otimes \varrho_{\mathrm{B}}\right] .
$$

We refer to the dimensionless operator $H_{\chi}$ as the correlation parent operator. The equivalent correlating transformation in this regime is obtained by inserting Eq. (2) into Eq. (1) as $\varrho_{\mathrm{SB}}=\mathscr{E}_{\chi}\left[\varrho_{\mathrm{S}} \otimes \varrho_{\mathrm{B}}\right]:=\varrho_{\mathrm{S}} \otimes \varrho_{\mathrm{B}}-i\left[H_{\chi}, \varrho_{\mathrm{S}} \otimes \varrho_{\mathrm{B}}\right]$.

Correlating transformation for a general state.-In the above scenario, we applied a known unitary transformation to create a correlated state and obtained the correlation $\chi$ in terms of the input uncorrelated state $\varrho_{\mathrm{S}} \otimes \varrho_{\mathrm{B}}$. Now, we return to our question of interest: to find the operation that transforms a given $\varrho_{\mathrm{S}} \otimes \varrho_{\mathrm{B}}$ to its associated $\varrho_{\mathrm{SB}}$ with a definite correlation operator $\chi$ that is not necessarily small.

Although for any given pair of quantum states $Q_{1}$ and $\varrho_{2}$, it is possible to find a quantum map or channel $\mathscr{E}$ such that $\mathscr{E}\left[\varrho_{1}\right]=\varrho_{2}$ [36], such a map does not necessarily have a unitary representation. Hence, if we postulate the form given in Eq. (2) as the equation connecting $\varrho_{\mathrm{S}} \otimes \varrho_{\mathrm{B}}$ and $\chi$, for some $H_{\chi}$, it generally does not have a Hermitian solution for $H_{\chi}$. However, with the insight gained from the weakly correlated case, we still choose $\chi$ as in Eq. (2) but relax the Hermiticity constraint on $H_{\chi}$. Since the left side of Eq. (2) is Hermitian, to ensure the Hermiticity of the right side, with a non-Hermitian $H_{\chi}$, we introduce a generalized commutator $\llbracket A, B \rrbracket=A B-B^{\dagger} A^{\dagger}$ [37] and replace Eq. (2) with

$$
\chi=-i \llbracket H_{\chi}, \varrho_{\mathrm{S}} \otimes \varrho_{\mathrm{B}} \rrbracket .
$$


We consider this relation as the general definition of $H_{\chi}$, valid for any $\chi$. Fortunately, this equation always has a solution for $H_{\chi}$, provided that $P_{0}(\tau) \chi(\tau) P_{0}(\tau)=0$, where $P_{0}(\tau)$ is the projector onto the null space of $\varrho_{\mathrm{S}}(\tau) \otimes \varrho_{\mathrm{B}}(\tau)$ [38]. We prove that this condition is always satisfied [39] and we also provide the solution for $H_{\chi}$. Using Eq. (3), we define our correlating transformation $\mathscr{E}_{\chi}$ as

$\varrho_{\mathrm{SB}}=\mathscr{E}_{\chi}\left[\varrho_{\mathrm{S}} \otimes \varrho_{\mathrm{B}}\right]:=\varrho_{\mathrm{S}} \otimes \varrho_{\mathrm{B}}-i \llbracket H_{\chi}, \varrho_{\mathrm{S}} \otimes \varrho_{\mathrm{B}} \rrbracket$.

We note that neither the solution of Eq. (3) for $H_{\chi}$ nor the formal connection of $\chi$ to $\varrho_{\mathrm{S}} \otimes \varrho_{\mathrm{B}}$ is unique (one may suggest other forms), which means that there is a kind of gauge freedom in choosing the correlating transformation. However, this nonuniqueness does not affect our formalism because any solution of Eq. (3) and any properly chosen form for $\mathscr{E}_{\chi}$ must generate the same given $\chi$-which is the physical quantity that should remain unchanged under these gauge transformations.

Note that the uncorrelated state $\varrho_{S} \otimes \varrho_{\mathrm{B}}$ is not the state of the total system (because, in general, $\chi \neq 0$ ); rather, we take this state as the description of the total system in a pertinent correlation picture. In order to keep the dynamics of the state in this picture faithful to the Schrödinger equation, we need to devise an appropriate formulation. Figure 1 depicts the correlating transformation and the associated correlation picture-which is explained in detail below.

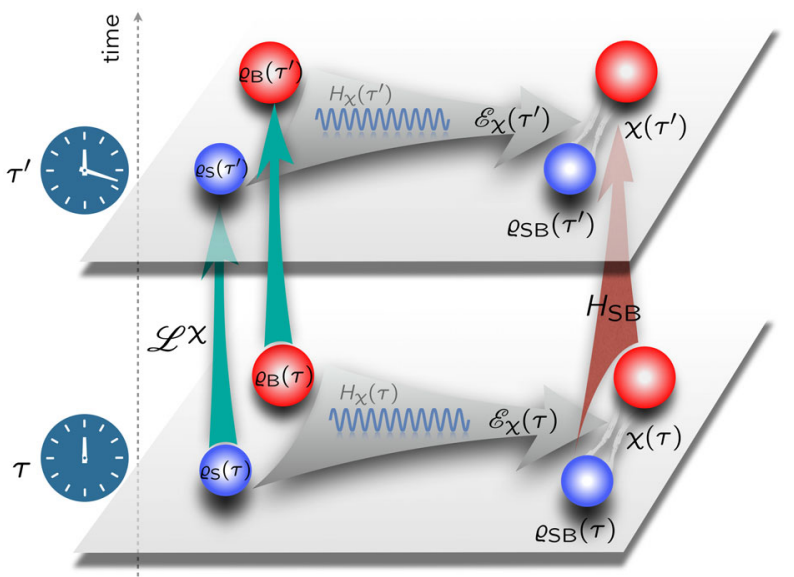

FIG. 1. Description of the correlation picture. At any time $\tau$ (or $\tau^{\prime}$ ), a correlating transformation $\mathscr{E}_{\chi}$ transforms an uncorrelated state $\varrho_{\mathrm{S}} \otimes \varrho_{\mathrm{B}}$ to a correlated state $\varrho_{\mathrm{SB}}=\varrho_{\mathrm{S}} \otimes \varrho_{\mathrm{B}}+\chi$, at the same instant of time, due to an abstract correlation-dependent parent operator given by $H_{\chi}$. Using this transformation, we obtain the temporal evolution of the uncorrelated system with a universal Lindblad-like generator $\mathscr{L}^{\chi}$ [see Eq. (9)] constructed from $H_{\mathrm{SB}}$, the generator of the total system dynamics in the Schrödinger picture.

\section{CORRELATION-PICTURE DYNAMICS}

We aim to apply our correlation-picture transformation between the correlated and uncorrelated states, $\varrho_{\mathrm{SB}}$ and $\varrho_{\mathrm{S}} \otimes \varrho_{\mathrm{B}}$, respectively, to obtain a dynamical equation for $\varrho_{S}$. Our approach can be considered in the spirit of the derivation of the Nakajima-Zwanzig (NZ) equation $[5,6]$. However, rather than applying a decorrelating projector to omit system-bath correlations (while implicitly carrying correlations into another complementary equation), we employ our correlating transformation within the Schrödinger equation of the total system. Hence, we explicitly retain contributions to the system dynamics from the correlations in the total system.

Let us assume that the total Hamiltonian of the system and the bath is given by $H_{\mathrm{SB}}=H_{\mathrm{S}}+H_{\mathrm{B}}+H_{\mathrm{I}}$, where the last term denotes the system-bath interaction. We employ the correlating transformation (4) to obtain a counterpart for the Schrödinger picture generator $\mathscr{D}_{3}[\circ]=-i\left[H_{\mathrm{SB}}, \circ\right]$ (throughout this paper, we assume the dimensionless units $\hbar \equiv k_{\mathrm{B}} \equiv 1$ ). More precisely, we define this operator $\mathscr{D}_{\odot}$ such that

$$
\mathscr{D}_{\jmath}\left[\varrho_{\mathrm{SB}}(\tau)\right]=\mathscr{D}_{c}\left[\varrho_{\mathrm{S}}(\tau) \otimes \varrho_{\mathrm{B}}(\tau)\right] .
$$

By inserting the correlating transformation (4) in the Schrödinger equation as $\mathscr{D}_{s}\left[\varrho_{\mathrm{SB}}(\tau)\right]=\mathscr{D}_{3}\left\{\mathscr{C}_{\chi}\left[\varrho_{\mathrm{S}}(\tau) \otimes\right.\right.$ $\left.\left.\varrho_{\mathrm{B}}(\tau)\right]\right\}$, we obtain the correlation-picture generator as

$$
\mathscr{D}_{c}[\circ]=-i\left[H_{\mathrm{SB}}, \circ\right]-\left[H_{\mathrm{SB}}, \llbracket H_{\chi}, \circ \rrbracket\right] .
$$

Although the dynamics described by $\mathscr{D}_{e}$ is fully equivalent to the Schrödinger picture dynamics governed by $\mathscr{D}_{j}$, working in the correlation picture offers clear advantages.

\section{UNIVERSALITY OF THE LINDBLAD-LIKE FORM FOR OPEN-SYSTEM DYNAMICS}

We show in the following that working in the correlation picture leads to a ULL equation. In the next sections, we discuss applications and further properties of the ULL formalism.

\section{A. General theory}

From Eq. (6), we can readily obtain the dynamics of the subsystem by tracing over the bath degrees of freedom in $\dot{\varrho}_{\mathrm{SB}}(\tau)=\mathscr{D}_{c}\left[\varrho_{\mathrm{S}}(\tau) \otimes Q_{\mathrm{B}}(\tau)\right]$. To show that the subsystem dynamics has a Lindblad-like form, we use the expansions of $H_{\mathrm{I}}=\sum_{i=1}^{d_{\mathrm{s}}^{2}-1} \mathcal{S}_{i} \otimes \mathscr{B}_{i} \quad$ and $H_{\chi}(\tau)=$ $\sum_{j=0}^{d_{\mathrm{S}}^{2}-1} \mathcal{S}_{j} \otimes \mathscr{B}_{j}^{\chi}(\tau)$, where $\left\{\mathcal{S}_{i}\right\}_{i=1}^{d_{\mathrm{S}}^{2}-1}$ is the basis of Hermitian operators defined on the system Hilbert space. Here, $d_{\mathrm{S}}$ is the dimension of the Hilbert space of the system, and $\delta_{0}=\mathbb{I}$. We emphasize the difference between the operators $\mathscr{B}_{i}$ and $\mathscr{B}_{i}^{\chi}(\tau)$, which are related to $H_{\mathrm{I}}$ and $H_{\chi}$, 
respectively. Inserting these expansions into $\dot{\varrho}_{\mathrm{S}}=$ $\operatorname{Tr}_{\mathrm{B}}\left[-i\left[H_{\mathrm{SB}}, \varrho_{\mathrm{S}} \otimes \varrho_{\mathrm{B}}\right]-\left[H_{\mathrm{SB}}, \llbracket\left[H_{\chi}, \varrho_{\mathrm{S}} \otimes \varrho_{\mathrm{B}} \rrbracket\right]\right]\right.$ yields

$$
\begin{aligned}
\dot{\varrho}_{\mathrm{S}}= & -i\left[H_{\mathrm{S}}+\operatorname{Tr}_{\mathrm{B}}\left[H_{\mathrm{I}} \varrho_{\mathrm{B}}\right], \varrho_{\mathrm{S}}\right] \\
& -\sum_{i \neq 0, j}\left[\mathcal{S}_{i}, c_{i j} \mathcal{\delta}_{j} \varrho_{\mathrm{S}}-c_{i j}^{*} \varrho_{\mathrm{S}} \mathcal{\delta}_{j}\right],
\end{aligned}
$$

where

$$
c_{i j}(\tau)=\left\langle\mathscr{B}_{i} \mathscr{B}_{j}^{\chi}(\tau)\right\rangle_{\mathrm{B}}
$$

are the elements of the covariance matrix $C(\tau)$ of the bath operators (with $\langle\circ\rangle_{\mathrm{B}}=\operatorname{Tr}_{\mathrm{B}}\left[\varrho_{\mathrm{B}}(\tau) \circ\right]$ ). Here, unlike the standard Lindblad equation, these bath operators are obtained not only from the interaction Hamiltonian but also from the correlation parent operator $H_{\chi}$ defined in Eq. (3). We rewrite $C(\tau)$ in Eq. (7) in terms of its Hermitian and anti-Hermitian parts as $C(\tau)=A(\tau)+i B(\tau)$, with Hermitian matrices $A(\tau)=\left[a_{i j}(\tau)\right]$ defined by $a_{i j}(\tau)=$ $(1 / 2)\left[C(\tau)+C^{\dagger}(\tau)\right]_{i j}$ and $B(\tau)=\left[b_{i j}(\tau)\right]$ defined by $b_{i j}(\tau)=(-i / 2)\left[C(\tau)-C^{\dagger}(\tau)\right]_{i j}$, for $i, j \geq 1$. This process leads to an exact Lindblad-like master equation for the system,

$$
\begin{aligned}
\dot{\varrho}_{\mathrm{S}} & =\mathscr{L}^{\chi}\left[\varrho_{\mathrm{S}}\right] \\
& =-i\left[H_{\mathrm{S}}+\mathbb{L}_{\mathrm{L}}^{\chi}, \varrho_{\mathrm{S}}\right]+\sum_{m} \gamma_{m}^{\chi}\left(2 L_{m}^{\chi} \varrho_{\mathrm{S}} L_{m}^{\chi^{\dagger}}-\left\{L_{m}^{\chi^{\dagger}} L_{m}^{\chi}, \varrho_{\mathrm{S}}\right\}\right),
\end{aligned}
$$

where $\{X, Y\} \equiv X Y+Y X$ denotes the anticommutator. In this equation, the quasirates $\gamma_{m}^{\chi}$ are the eigenvalues of the matrix $A(\tau)$. The jump operators are given by $L_{m}^{\chi}=\sum_{j \neq 0} V_{m j} \mathcal{S}_{j}$, where $\left\{V_{m j}\right\}_{j}$ are the elements of the eigenvector corresponding to the eigenvalue $\gamma_{m}^{\chi}$. Furthermore, the Lamb-shift-like Hamiltonian $\ln _{\mathrm{L}}^{\chi}(\tau)=$ $\left\langle H_{\mathrm{I}}\right\rangle_{\mathrm{B}}+2 \sum_{i \neq 0} \operatorname{Im}\left(c_{i 0}(\tau)\right) \mathcal{S}_{i}+\sum_{i \neq 0, j \neq 0} b_{i j}(\tau) \mathcal{S}_{i} \mathcal{S}_{j}$, where $b_{i j}$ are the elements of the matrix $B(\tau)$. For more details on the derivation, see Ref. [39].

Several remarks are in order here:

(i) Although by combining known results in the literature one may infer time-local Lindblad-like forms for the dynamics under the assumptions of linearity or absence of initial system-environment correlations $[3,15,17]$, the ULL equation (9) is completely general; we have made no assumptions on the initial system-environment correlations or the strength of the system-environment interaction. In addition, the ULL equation is built explicitly on a microscopic theory of correlations in the total system.

(ii) Unlike the well-known Markovian embedding, where a non-Lindblad (non-Markovian) evolution is mapped to a Lindblad (Markovian) evolution for a specific larger system employing an ancillary system [40], we have proven here that the Lindbladlike form is derived for the open-system dynamics itself.

(iii) We note that the coefficients in the ULL master equation (8) refer to the correlation functions $\left\langle\mathscr{B}_{i} \mathscr{B}_{j}^{\chi}(\tau)\right\rangle_{\mathrm{B}}$, defined by the correlation parent operator and are thus different from the conventional correlation functions of bath operators $\left\langle\mathscr{B}_{i}(\tau) \mathscr{B}_{j}(\tau)\right\rangle_{\mathrm{B}}$ appearing in the standard Markovian Lindblad equation [3], where the average is taken on a constant bath state, and $\mathscr{B}_{i}(\tau)=U_{\mathrm{B}}^{\dagger}(\tau) \mathscr{B}_{i} U_{\mathrm{B}}(\tau)$ [with $U_{\mathrm{B}}(\tau)=e^{-i \tau H_{\mathrm{B}}}$ ].

(iv) Since $\mathscr{L}^{x}$ depends on the state of the system, Eq. (9) is formally a nonlinear equation. Indeed, the linearity constraint on the full dynamics of quantum systems does not imply a similar restriction on the dynamics of a subsystem, and this nonlinearity is naturally expected for a general dynamical equation. Nevertheless, we show in Ref. [39] that our ULL master equation (9) is linear in two important cases: (a) if there is no initial correlation, i.e., $\chi(0)=0$, where we show that $\chi(\tau)$ can be explicitly expressed in terms of the system-bath product state, and (b) if the domain of $\mathscr{L}^{x}$ is restricted to a set of states $\left\{\varrho_{\mathrm{S}}^{(i)}\right\}$ forming a convex decomposition of the state of the system, i.e., $\varrho_{\mathrm{S}}=\sum_{i} p_{i} \varrho_{\mathrm{S}}^{(i)}$ (but here, the initial total state may be correlated).

\section{B. Example I: Jaynes-Cummings model with initial correlation}

To illustrate universality of the dynamical equation (9), even in the presence of initial system-bath correlations, we begin with a proof-of-principle example, the well-known, exactly solvable, Jaynes-Cummings model [41], and show that the dynamics of the two-level system is described by the ULL equation even when the system is correlated with a bosonic mode.

Consider a two-level system interacting with a single bosonic mode under the Jaynes-Cummings Hamiltonian. The system Hamiltonian is $H_{\mathrm{S}}=\left(\omega_{0} / 2\right) \sigma_{z}$, where $\sigma_{ \pm}=$ $\left(\sigma_{x} \pm i \sigma_{y}\right) / 2$ and $\sigma_{x}, \sigma_{y}$, and $\sigma_{z}$ are the $x, y$, and $z$ Pauli operators, respectively; the bosonic bath Hamiltonian is $H_{\mathrm{B}}=\omega \hat{a}^{\dagger} \hat{a}$, where $\hat{a}^{\dagger}(\hat{a})$ is the creation (annihilation) operator of the bosonic mode; and $H_{\mathrm{I}}=\lambda\left(\sigma_{+} \otimes \hat{a}+\sigma_{-} \otimes\right.$ $\left.\hat{a}^{\dagger}\right)$ describes the system-bath interaction. For simplicity, we assume that $\omega_{0}=\omega$ and that the initial state of the total system is in a correlated state $|\psi(0)\rangle=r_{1}|\mathrm{e}, 0\rangle+r_{2}|\mathrm{~g}, 1\rangle$, where both $r_{1}$ and $r_{2}$ are real numbers. Choosing $\mathcal{S}_{0}=\mathbb{I} / \sqrt{2}, \quad \mathcal{S}_{1}=\sigma_{x} / \sqrt{2}, \quad \mathcal{S}_{2}=\sigma_{y} / \sqrt{2}, \quad$ and $\quad \mathcal{S}_{3}=$ $\sigma_{z} / \sqrt{2}$ as the basis operators, we can find $\left\{\mathscr{B}_{i}^{\chi}\right\}_{i}$ (see Ref. [39]). Thus, the bath covariances are obtained as $c_{10}=c_{20}=0, c_{11}=c_{22}=\lambda\left(-2 i r_{1} r_{2}+\alpha_{1} \alpha_{2}\right) /\left(2 \alpha_{1}^{2}-2\right)$, and $c_{12}=-c_{21}=\lambda\left(2 r_{1} r_{2} \alpha_{1}+i \alpha_{2}\right) /\left(2\left(1-\alpha_{1}^{2}\right)\right)$, where 
$\alpha_{1}=\left(1-2 r_{1}^{2}\right) \cos (2 \lambda \tau) \quad$ and $\quad \alpha_{2}=\left(1-2 r_{1}^{2}\right) \sin (2 \lambda \tau)$. Following the steps of the derivation of Eq. (9), we obtain

$$
\begin{aligned}
\dot{\varrho}_{\mathrm{S}}= & -i\left[H_{\mathrm{S}}+\tilde{\omega}_{0} \sigma_{z}, \varrho_{\mathrm{S}}\right]+\gamma_{1}^{\chi}\left(2 \sigma_{-} \varrho_{\mathrm{S}} \sigma_{+}-\left\{\sigma_{+} \sigma_{-}, \varrho_{\mathrm{S}}\right\}\right) \\
& -\gamma_{2}^{\chi}\left(2 \sigma_{+} \varrho_{\mathrm{S}} \sigma_{-}-\left\{\sigma_{-} \sigma_{+}, \varrho_{\mathrm{S}}\right\}\right),
\end{aligned}
$$

where $\quad \tilde{\omega}_{0}=4 \lambda r_{1} r_{2} \alpha_{1} /\left(1+4 r_{1}^{2}-4 r_{1}^{4}-\left(\alpha_{1}^{2}-\alpha_{2}^{2}\right)\right)$, $\gamma_{1}^{\chi}=-\lambda \alpha_{2} /\left(2\left(1-\alpha_{1}\right)\right)$, and $\gamma_{2}^{\chi}=\lambda \alpha_{2} /\left(2\left(1+\alpha_{1}\right)\right)$ [39]. We emphasize that this equation is in the ULL form and is valid even with initial system-bath correlations.

\section{REDUCTION TO A MARKOVIAN EQUATION}

Based on our general dynamical equation where systembath correlations are fully incorporated, we can obtain simpler expressions for the case where the correlations are small. This approach is valid, e.g., in the vicinity of time instants at which the correlation vanishes or becomes negligible. In other words, we introduce a weak-correlation approximation. In such cases, we can simplify our ULL master equation into a MLL master equation, in which jump rates are positive-as expected from Markovian dynamics [9]. Below, we show that this equation correctly characterizes the universal quadratic short-time behavior of the system dynamics where the standard Lindblad master equation may fail $[24,25]$.

We assume that at $\tau_{0}$ the correlation vanishes. Without loss of generality, we take $\tau_{0}=0$; thus, $\chi(0)=0$. We allow the correlations to accumulate in the subsequent time steps due to the dynamics. To first order in the time argument $\tau$, we find that the correlation satisfies Eq. (3) with $H_{\chi}(\tau)=\tau \tilde{H}_{\mathrm{I}}(\tau)$, where $\tilde{H}_{\mathrm{I}}(\tau)=\sum_{i \neq 0} \mathcal{S}_{i} \otimes\left(\mathscr{B}_{i}-\left\langle\mathscr{B}_{i}\right\rangle_{\mathrm{B}}\right)-\sum_{i \neq 0}\left\langle\delta_{i}\right\rangle_{\mathrm{S}} \mathscr{B}_{i}$ and $\langle\circ\rangle_{\mathrm{S}}=\operatorname{Tr}_{\mathrm{S}}\left[\varrho_{\mathrm{S}}(\tau) \circ\right]$. Thus, from the knowledge of $H_{\chi}$, we can read $\mathscr{B}_{j}^{\chi}(\tau)=\tau\left(\mathscr{B}_{j}-\left\langle\mathscr{B}_{j}\right\rangle_{\mathrm{B}}\right)$, where $j \geq 1$. Substituting these expressions into Eq. (8), the bath covariance matrix becomes

$$
c_{i j}(\tau)=\tau \operatorname{Cov}_{\mathrm{B}}\left(\mathscr{B}_{i}, \mathscr{B}_{j}\right), \quad i, j \geq 1,
$$

where $\operatorname{Cov}_{\mathrm{B}}\left(O_{1}, O_{2}\right)=\left\langle O_{1} O_{2}\right\rangle_{\mathrm{B}}-\left\langle O_{1}\right\rangle_{\mathrm{B}}\left\langle O_{2}\right\rangle_{\mathrm{B}}$. Since the covariance matrix $C(\tau)$ is positive-semidefinite, $a_{i j}(\tau)=$ $c_{i j}(\tau)$ and $b_{i j}(\tau)=0$. The positivity of $A$ implies positivity of the rates $\gamma_{m}^{\chi} \geq 0$, which is a necessary feature of Markovian dynamical evolution. To obtain an equation with no dependence on the state of the bath [recall that $\ln _{\mathrm{L}}^{\chi}(\tau)$ and $c_{i j}(\tau)$ depend on $\varrho_{\mathrm{B}}(\tau)$ ], we also expand $\varrho_{\mathrm{B}}(\tau)$ around $\tau_{0}=0$ and keep relevant terms up to the first order in $\tau$. Thus, we obtain

$$
\begin{aligned}
a_{i j}(\tau) \approx & \tau \operatorname{Cov}_{\mathrm{B}_{0}}\left(\mathscr{B}_{i}, \mathscr{B}_{j}\right) ; \quad b_{i j}(\tau)=0 \\
\mathbb{L}_{\mathrm{L}}^{\chi}(\tau) \approx & \left\langle H_{\mathrm{I}}\right\rangle_{\mathrm{B}_{0}}-i \tau\left\langle\left[H_{\mathrm{I}}, \tilde{H}_{\mathrm{B}}\right]\right\rangle_{\mathrm{B}_{0}} \\
& -2 \tau \sum_{(i, j) \neq(0,0)}\left\langle\mathcal{S}_{j}\right\rangle_{\mathrm{S}_{0}} \operatorname{Im}\left\langle\mathscr{B}_{i} \mathscr{B}_{j}\right\rangle_{\mathrm{B}_{0}} \mathcal{S}_{i},
\end{aligned}
$$

where subscripts $B_{0}$ and $S_{0}$ indicate that the averages or covariances are taken with respect to $\varrho_{\mathrm{B}}(0)$ and $\varrho_{\mathrm{S}}(0)$ rather than $\varrho_{\mathrm{B}}(\tau)$ and $\varrho_{\mathrm{S}}(\tau)$. In Eq. (12), we have defined $\tilde{H}_{\mathrm{B}}=$ $H_{\mathrm{B}}+\left\langle H_{\mathrm{I}}\right\rangle_{\mathrm{S}_{0}}$ (see Ref. [39] for more details). Equation (9)bearing in mind Eq. (12)—describes the short-time Markovian dynamics around a point of vanishing correlation.

We emphasize that our weak-correlation assumption is exact up to the first order in $\tau$. If we extend this Markovian dynamical equation to longer times, it may still work as an approximation for the exact dynamics, e.g., when the correlation repeatedly becomes zero [18,39]. Although, at first sight, expanding around a point of vanishing correlation may seem equivalent to the standard Born approximation, we illustrate in the next example that the MLL equation can be different from the Redfield equation. In addition, unlike the Redfield equation $[3,4]$, the MLL equation always keeps the state positive, hence avoiding the so-called slippage issue that afflicts the Redfield equation $[42,43]$.

A final remark regarding the applicability of the MLL approximation in other regimes is in order. If the system has an asymptotic state, a candidate for such a state can be $\varrho^{\star}=\sum_{n}\left\langle E_{n}\left|\varrho_{\mathrm{SB}}(0)\right| E_{n}\right\rangle\left|E_{n}\right\rangle\left\langle E_{n}\right|$, where $\left\{\left|E_{n}\right\rangle\right\}$ are the eigenvectors of $H_{\mathrm{SB}}$ [39]. Now assume that (i) the system is strongly interacting with the bath (i.e., $H_{\mathrm{I}}$ is the dominant term in the total Hamiltonian such that $H_{\mathrm{SB}} \approx H_{\mathrm{I}}$ ), (ii) the interaction Hamiltonian contains only one term, $H_{\mathrm{I}}=\mathcal{S} \otimes \mathscr{B}$, and (iii) the initial state of the system and the bath is uncorrelated, $\chi(0)=0$. It is straightforward to show that under these conditions, $Q^{\star}$ is an uncorrelated state, and thus, also in this case, a MLL equation describes the asymptotic dynamics [39]. In the next section, we provide another case where the MLL approximation also holds, and later we illustrate these behaviors with two examples.

\section{DYNAMICS OF THE CORRELATION: SYSTEMATIC WEAK-CORRELATION EXPANSION}

The above MLL approximation shows that the correlation picture and the ULL equation can offer far-reaching practical implications beyond their fundamental appeal. We identify that the weak-correlation approximation is the basic ingredient of the MLL equation. Expanding upon this is desirable as it can make the ULL methodology more amenable to practical investigations of a diverse set of systems.

\section{A. General theory}

To go further and demonstrate that the ULL equation systematically enables such a rich approximative structure, in the following, we develop a perturbative weak-correlation expansion for the ULL equation. In particular, by using the MLL toolbox as a starting point for a perturbative expansion of the correlation matrix in the interaction 
picture, we find an exact dynamical equation for the correlation $\chi$ (boldface denotes the interaction picture) and expand it in terms of the interaction Hamiltonian $\boldsymbol{H}_{\mathrm{I}}$ as

$$
\chi(\tau)=\sum_{\ell=0}^{\infty} \boldsymbol{f}_{\ell}(\tau)
$$

where $\boldsymbol{f}_{0}(\tau) \equiv \chi(0)$ and $\boldsymbol{f}_{\ell}(\tau)$ is of the order of $\boldsymbol{H}_{\mathrm{I}}^{\ell}$, for $\ell \geq 1$. We incorporate the first $\ell+1$ terms within this expansion in the ULL dynamical equations for the system and the bath and derive the associated $\ell$ th-order approximate ULL equations, referred to as the "ULL $\ell$ " equations.

Let us provide an outline of the weak-correlation expansion-for details, see Ref. [39]. We start by the decomposition of the state of the total system as in Eq. (1). The total system at a later time $\tau+\xi$ is given by

$$
\varrho_{\mathrm{SB}}(\tau+\xi)=U_{\xi} \varrho_{\mathrm{S}}(\tau) \otimes \varrho_{\mathrm{B}}(\tau) U_{\xi}^{\dagger}+U_{\xi} \chi(\tau) U_{\xi}^{\dagger},
$$

where $U_{\xi}=e^{-i H_{\mathrm{SB}} \xi}$. The first term of the above equation represents the evolution of an uncorrelated state $\varrho_{\mathrm{S}}(\tau) \otimes \varrho_{\mathrm{B}}(\tau)$, which up to the first order in $\xi$, is given by the MLL dynamical equation. Using Eq. (14) and the definition of the correlation operator (1) at time $\tau+\xi$, one obtains, after some algebra [39],

$$
\dot{\chi}(\tau)=-i\left[\tilde{\boldsymbol{H}}_{\mathrm{I}}(\tau), \boldsymbol{\varrho}_{\mathrm{S}}(\tau) \otimes \varrho_{\mathrm{B}}(\tau)\right]+\mathcal{Y}_{\tau}[\chi(\tau)],
$$

where we have defined

$$
\begin{aligned}
\mathcal{Y}_{\tau}[\circ]:= & -i\left[\boldsymbol{H}_{\mathrm{I}}(\tau), \circ\right]+i \operatorname{Tr}_{\mathrm{B}}\left[\boldsymbol{H}_{\mathrm{I}}(\tau), \circ\right] \otimes \boldsymbol{\varrho}_{\mathrm{B}}(\tau) \\
& +i \boldsymbol{\varrho}_{\mathrm{S}}(\tau) \otimes \operatorname{Tr}_{\mathrm{S}}\left[\boldsymbol{H}_{\mathrm{I}}(\tau), \circ\right] .
\end{aligned}
$$

Using integration and iterations, a solution to Eq. (15) is obtained as

$$
\begin{aligned}
\chi(\tau)= & \sum_{k=0}^{\infty} \int_{0}^{\tau} d s_{1} \int_{0}^{s_{1}} d s_{2} \ldots \int_{0}^{s_{k-1}} d s_{k} \mathcal{Y}_{s_{1}}\left[\mathcal{Y}_{s_{2}} \ldots\left[\mathcal{Y}_{s_{k}}[\chi(0)]\right] \ldots\right] \\
& +\sum_{k=0}^{\infty} \int_{0}^{\tau} d s_{1} \int_{0}^{s_{1}} d s_{2} \ldots \int_{0}^{s_{k-1}} d s_{k} \mathcal{Y}_{s_{1}}\left[\mathcal{Y}_{s_{2}} \ldots\left[\mathcal{Y}_{s_{k}}\left[-i \int_{0}^{s_{k}} d s\left[\tilde{\boldsymbol{H}}_{\mathrm{I}}(s), \boldsymbol{\varrho}_{\mathrm{S}}(s) \otimes \boldsymbol{\varrho}_{\mathrm{B}}(s)\right]\right]\right] \ldots\right],
\end{aligned}
$$

which is symbolically in the form of Eq. (13). Starting from Eq. (17), we can systematically approximate the correlation and hence the ULL dynamical equation. If the initial system-bath correlation vanishes, the first-order approximation (with respect to $\tilde{\boldsymbol{H}}_{\mathrm{I}}$ ) gives

$$
\chi^{(1)}(\tau)=-i \int_{0}^{\tau} d s\left[\tilde{\boldsymbol{H}}_{\mathrm{I}}(s), \boldsymbol{\varrho}_{\mathrm{S}}(s) \otimes \boldsymbol{\varrho}_{\mathrm{B}}(s)\right]
$$

Using this relation to derive the correlation parent operator [39], one can obtain a weak-correlation approximation for the ULL dynamical equations for the system and the bath,

$$
\begin{aligned}
\dot{\boldsymbol{\varrho}}_{\mathrm{S}}(\tau)= & -i\left[\operatorname{Tr}_{\mathrm{B}}\left[\boldsymbol{H}_{\mathrm{I}}(\tau) \boldsymbol{\varrho}_{\mathrm{B}}(\tau)\right], \boldsymbol{\varrho}_{\mathrm{S}}(\tau)\right] \\
& -\operatorname{Tr}_{\mathrm{B}}\left[\boldsymbol{H}_{\mathrm{I}}(\tau), \int_{0}^{\tau} d s\left[\tilde{\boldsymbol{H}}_{\mathrm{I}}(s), \boldsymbol{\varrho}_{\mathrm{S}}(s) \otimes \boldsymbol{\varrho}_{\mathrm{B}}(s)\right]\right], \\
\dot{\boldsymbol{\varrho}}_{\mathrm{B}}(\tau)= & -i\left[\operatorname{Tr}_{\mathrm{S}}\left[\boldsymbol{H}_{\mathrm{I}}(\tau) \boldsymbol{\varrho}_{\mathrm{S}}(\tau)\right], \boldsymbol{\varrho}_{\mathrm{B}}(\tau)\right] \\
& -\operatorname{Tr}_{\mathrm{S}}\left[\boldsymbol{H}_{\mathrm{I}}(\tau), \int_{0}^{\tau} d s\left[\tilde{\boldsymbol{H}}_{\mathrm{I}}(s), \boldsymbol{\varrho}_{\mathrm{S}}(s) \otimes \boldsymbol{\varrho}_{\mathrm{B}}(s)\right]\right],
\end{aligned}
$$

which are of second order with respect to $\tilde{\boldsymbol{H}}_{\mathrm{I}}$ - hence, we refer to them as the ULL2 equations. Returning to the Schrödinger picture, we need to solve these coupled differential equations for $\varrho_{\mathrm{S}}(\tau)$ and $\varrho_{\mathrm{B}}(\tau)$ in a self-consistent fashion, to obtain approximate states of the system and the bath-see Ref. [39] for details and a discussion on the numerical integration of the ULL2 equations.

It is instructive to compare the system ULL2 equation (19) with the system second-order NZ (NZ2) equation $[3,4]$,

$$
\begin{aligned}
\dot{\boldsymbol{\varrho}}_{\mathrm{S}}(\tau)= & -i\left[\operatorname{Tr}_{\mathrm{B}}\left[\boldsymbol{H}_{\mathrm{I}}(\tau) \boldsymbol{\varrho}_{\mathrm{B}}(0)\right], \boldsymbol{\varrho}_{\mathrm{S}}(\tau)\right] \\
& -\operatorname{Tr}_{\mathrm{B}}\left[\boldsymbol{H}_{\mathrm{I}}(\tau), \int_{0}^{\tau} d s\left[\boldsymbol{H}_{\mathrm{I}}(s), \boldsymbol{\varrho} \boldsymbol{S}(s) \otimes \boldsymbol{\varrho}_{\mathrm{B}}(0)\right]\right] .
\end{aligned}
$$

These equations appear rather similar, but there are two key differences: (i) The state of the bath is time dependent in the ULL2 equation, whereas in the NZ2 equation, it is a constant state, which is usually assumed to be the initial or equilibrium state of the bath, and (ii) in the integral of the ULL2 equation rather than $\boldsymbol{H}_{\mathrm{I}}(s)$, we have its effective form $\tilde{\boldsymbol{H}}_{\mathrm{I}}(s)$. These points suggest that the performance of the ULL2 equation is generally different from that of the NZ2 equation, and thereby one may expect that the ULL2 equation either performs similarly to the NZ2 equation or outperforms it. We illustrate the validity of this observation later in Sec. VIC. For comparison with several other methods, see Ref. [39]. 
For sufficiently short times, Eq. (18) reduces to

$$
\chi_{\mathrm{MLL}}^{(1)}(\tau) \approx-i \tau\left[\tilde{\boldsymbol{H}}_{\mathrm{I}}(\tau), \varrho_{\mathrm{S}}(\tau) \otimes \varrho_{\mathrm{B}}(\tau)\right]
$$

which is identical to the correlation operator obtained in the derivation of the MLL dynamical equation [39]. Hence, although in both ULL2 and the MLL equations $\chi$ is of the first order with respect to $\tilde{\boldsymbol{H}}_{\mathrm{I}}$, the ULL2 equation can be expected to lead to an improvement over the performance of the MLL approximation [39].

Let us discuss another asymptotic-time case where the MLL approximation holds. This case underlines that the utility of the MLL approximation is not necessarily limited to the short-time dynamics. If (i) the interaction is sufficiently weak (weak-coupling or weak-correlation regime) and (ii) the subsystem dynamics reaches a steady state in a finite time, one can approximate $\varrho_{\mathrm{S}}(s) \otimes \varrho_{\mathrm{B}}(s)$ in Eq. (18) with the tensor product of subsystem steady states $\varrho_{\mathrm{S}}^{\star} \otimes \varrho_{\mathrm{B}}^{\star}$ and hence $\tilde{\boldsymbol{H}}_{\mathrm{I}}(s) \approx \tilde{\boldsymbol{H}}_{\mathrm{I}}^{\star}$ at most times, which yields the MLL approximation $\chi_{\mathrm{MLL}}^{(1), \star}(\tau) \approx-i \tau\left[\tilde{\boldsymbol{H}}_{\mathrm{I}}^{\star}, \boldsymbol{\varrho}_{\mathrm{S}}^{\star} \otimes \boldsymbol{\varrho}_{\mathrm{B}}^{\star}\right]$. Since Eq. (18) becomes exact in the weak-coupling limit and typical quantum systems reach their steady state in finite times [44-47], one can conclude that the MLL equation modified by the subsystem steady states may hold at long times for typical systems [39].

Applying additional approximations on $\chi^{(1)}$ by imposing time locality and also assuming that the bath state remains constant in time, i.e., approximating $\chi^{(1)} \approx$ $-i \int_{0}^{\tau} d s\left[\tilde{\boldsymbol{H}}_{\mathrm{I}}(s), \boldsymbol{\varrho}_{\mathrm{S}}(\tau) \otimes \varrho_{\mathrm{B}}(0)\right]$, yields a time-local ULL2 equation.

Systematic derivation of higher-order correlation terms up to $\chi^{(\ell-1)}$ yielding the ULL $\ell$ approximations is straightforward but algebraically heavy, and so we leave this for future work. For details, see Ref. [39]. However, similar to other approximate techniques, it usually suffices to consider the lowest-order ULL2 or, at most, a few lowest orders. For a comparison with other techniques, see also Ref. [39].

We emphasize that although the validity of the weakcorrelation expansion hinges on the strength of the interaction Hamiltonian, it shows clear differences with standard weak-coupling approximations $[3,4]$. In particular, we note that our expansion uses the correlation in a direct fashion as the key ingredient.

In the following, we illustrate our weak-correlation ULL method through two examples. In example II, we show that the MLL equation captures the exact dynamics with good accuracy and outperforms the standard Markovian Lindblad equation. In addition, the time-local ULL2 equation becomes tantamount to the second-order time-convolutionless (TCL2) dynamical equation for the system, which gives the exact dynamics for this example. We also compare our solutions with a coarse-graining (CG) method $[4,18,25,48-51]$, which fails to exceed the performance of the ULL2 solution. In example III, we show that $\chi^{(1)}$ of Eq. (18) leads to a significant improvement in predicting the dynamics of the system, whereas the TCL2 and other approximating techniques such as the CG method do not provide such accuracy.

\section{B. Example II: Qubit in a bosonic bath}

Let us consider a two-level system or qubit interacting with a many-mode bosonic bath initially in the thermal state $\varrho_{\mathrm{B}}^{\beta}=e^{-\beta \sum_{n} \omega_{n} \hat{a}_{n}^{\dagger} \hat{a}_{n}} / \operatorname{Tr}\left[e^{-\beta \sum_{n} \omega_{n} \hat{a}_{n}^{\dagger} \hat{a}_{n}}\right]$ at temperature $T=1 / \beta$. Here, $\hat{a}_{l}$ is the annihilation operator for mode $l$. The total Hamiltonian reads

$$
H_{\mathrm{SB}}=\omega_{0} \sigma_{+} \sigma_{-}+\sum_{n} \omega_{n} \hat{a}_{n}^{\dagger} \hat{a}_{n}-\sigma_{x} \otimes \mathcal{O}_{\mathrm{B}}
$$

where $\mathcal{O}_{\mathrm{B}}=\sum_{n} \kappa_{n}\left(\hat{a}_{n}+\hat{a}_{n}^{\dagger}\right)$. Assuming that the qubit at all times retains only a small correlation with the bath, we conclude that Eq. (12) applies, and we obtain the following master equation:

$\dot{\varrho}_{\mathrm{S}}(\tau)=-i\left[H_{\mathrm{S}}, \varrho_{\mathrm{S}}(\tau)\right]+\gamma(\tau)\left(\sigma_{x} \varrho_{\mathrm{S}}(\tau) \sigma_{x}-\varrho_{\mathrm{S}}(\tau)\right)$,

where $\gamma(\tau)=2 \tau \operatorname{Cov}_{\mathrm{B}_{0}}\left(\mathcal{O}_{\mathrm{B}}, \mathcal{O}_{\mathrm{B}}\right)$, and $\operatorname{Cov}_{\mathrm{B}_{0}}\left(\mathcal{O}_{\mathrm{B}}, \mathcal{O}_{\mathrm{B}}\right)=$ $\int_{0}^{\infty} d \omega J(\omega)(2 n(\beta, \omega)+1)$ is given in terms of a spectral density function $J(\omega)$ and the bosonic occupation number $n(\beta, \omega)=\left(e^{\beta \omega}-1\right)^{-1}$. Equation (24) describes pure dephasing in the eigenbasis of $\sigma_{x}$ and gives the population of the excited state of the qubit as

$$
\varrho_{\mathrm{ee}}(\tau)=1 / 2+\left(\varrho_{\mathrm{ee}}(0)-1 / 2\right) e^{-2 \tau^{2} \operatorname{Cov}_{\mathrm{B}_{0}}\left(\mathcal{O}_{\mathrm{B}}, \mathcal{O}_{\mathrm{B}}\right)} .
$$

The solution of the exact dynamics for this example has been provided in Ref. [23] for $\omega_{0}=0$ and under the assumption of an initial thermal state for the bath and an Ohmic spectral density for the couplings of the interaction Hamiltonian, $J(\omega)=\eta \omega\left(1+\omega^{2} / \omega_{c}^{2}\right)^{-2}$, where $\omega_{c}$ is the cutoff frequency and $\eta$ denotes the coupling strength between the system and the bath. This solution provides a convenient means of studying the accuracy of Eq. (25). As we argued in Sec. V, under the mentioned conditions, even in the highly strong-coupling regime, the MLL equation is exact in asymptotic time. In this example, $H_{\mathrm{I}}$ only has one term, $H_{\mathrm{S}}$ is set to zero by $\omega_{0}=0$, and, for the chosen spectral density and the uncorrelated initial state, all the conditions are satisfied. Hence, the MLL equation gives an exact prediction for the asymptotic state; see Fig. 2 .

Figure 2 shows the evolution of the excited-state population and compares our MLL and ULL2 methods with the Redfield equation (an equation obtained by applying only the weak-coupling and time-locality approximations on the exact dynamics [4]), the TCL2 master equation $[3,4]$, and the exact solution. In addition, to make a comparison with a CG dynamical equation, we used the 

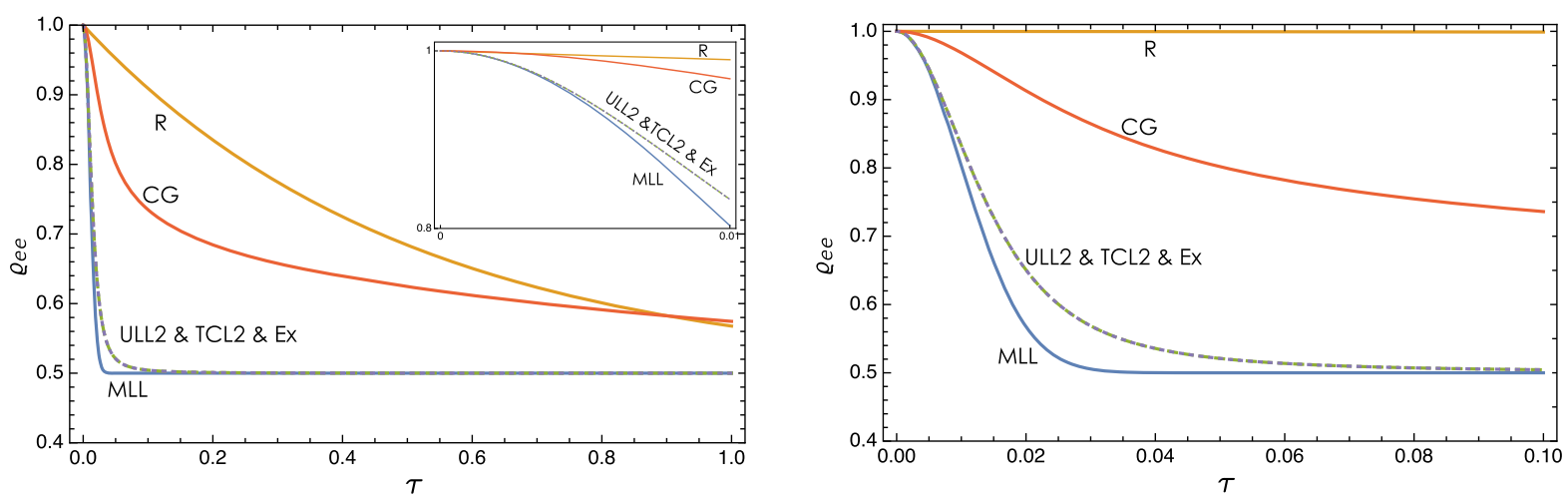

FIG. 2. A qubit in a bosonic bath. Left panel: Population of the excited state of the two-level system as a function of time for $\beta=1$, $\eta=0.5, \omega_{c}=100$, and $\omega_{0}=0$ (all in dimensionless units), where the two-level system is initially in the excited state. The labels TCL2 \& Ex, MLL, ULL2, CG, and R denote, respectively, the data from the second-order time-convolutionless (= Exact), MLL, time-local ULL2 (= Exact), coarse-graining, and the Redfield (= Markovian Lindblad) solutions. The inset is a magnified depiction of the population vs time for short times. Right panel: The same as the left panel but for $\beta=100$.

results of Ref. [50]; see Fig. 2. For this particular example, the time-local ULL2 dynamical equation is identical to the TCL2 dynamical equation [39], and both coincide with the exact dynamics. From this figure and the explicit form of the Redfield equation [39], it is clear that the Redfield equation is less accurate in the low-temperature limit. The MLL equation follows the exact solution relatively well, whereas the Redfield equation exhibits a relatively slower decay. Note that when $\omega_{0}=0$, the standard Lindblad equation is equivalent to the Redfield equation. For details of the derivation of the Redfield and the TCL2 equations and for the analysis of the short-time dynamics using the Lindblad-like model and the exact evolution, see Ref. [39].

In the following example, we illustrate that our Markovian approximation works well to describe the short-time dynamics in a system with non-Markovian features. We then show that the ULL2 equation outperforms other methods for later times and captures the longtime dynamics more accurately.

\section{Example III: Damped harmonic oscillator within a bath of oscillators}

Consider a quantum harmonic oscillator interacting with a bath of oscillators, with the total Hamiltonian given by

$H_{\mathrm{SB}}=\omega_{0} \hat{a}^{\dagger} \hat{a}+\sum_{k=1}^{M} \omega_{k} \hat{b}_{k}^{\dagger} \hat{b}_{k}+\sum_{k=1}^{M} g_{k}\left(\hat{a}^{\dagger} \hat{b}_{k}+\hat{a} \hat{b}_{k}^{\dagger}\right)$,

where $M$ is the number of the bath oscillators-see Fig. 3. For simplicity of the analysis, we assume the initial systembath state $\left(c_{0}|0\rangle+c_{1}|1\rangle\right)_{\mathrm{S}} \otimes|0\rangle_{\mathrm{B}}^{\otimes M}$, where $|i\rangle$ denotes the eigenstate of the corresponding number operator with eigenvalue $i$.

To obtain the MLL equation, we choose $\delta_{1}=\hat{a}+\hat{a}^{\dagger}$ and $\mathcal{S}_{2}=i\left(\hat{a}-\hat{a}^{\dagger}\right)$, and hence $\mathscr{B}_{1}=\sum_{k} g_{k}\left(\hat{b}_{k}+\hat{b}_{k}^{\dagger}\right) / 2$ and $\mathscr{B}_{2}=\sum_{k} i g_{k}\left(\hat{b}_{k}-\hat{b}_{k}^{\dagger}\right) / 2$. Inserting these into
Eq. (12) yields $a_{11}=a_{22}=\tau G / 4, a_{21}=a_{12}^{*}=i \tau G / 4$, $\operatorname{Im}\left\langle\mathscr{B}_{1} \mathscr{B}_{1}\right\rangle_{\mathrm{B}_{0}}=\operatorname{Im}\left\langle\mathscr{B}_{2} \mathscr{B}_{2}\right\rangle_{\mathrm{B}_{0}}=0$, and $\operatorname{Im}\left\langle\mathscr{B}_{2} \mathscr{B}_{1}\right\rangle_{\mathrm{B}_{0}}=$ $-\operatorname{Im}\left\langle\mathscr{B}_{1} \mathscr{B}_{2}\right\rangle_{\mathrm{B}_{0}}=G / 4$, where $G=\sum_{k}\left|g_{k}\right|^{2}$; hence, $\ln _{\mathrm{L}}^{\chi}=0$. The MLL equation thus reads

$$
\begin{aligned}
\dot{\varrho}_{\mathrm{S}}(\tau)= & -i\left[\omega_{0} \hat{a}^{\dagger} \hat{a}, \varrho_{\mathrm{S}}(\tau)\right]+G \tau\left(2 \hat{a} \varrho_{\mathrm{S}}(\tau) \hat{a}^{\dagger}-\hat{a}^{\dagger} \hat{a} \varrho_{\mathrm{S}}(\tau)\right. \\
& \left.-\varrho_{\mathrm{S}}(\tau) \hat{a}^{\dagger} \hat{a}\right) .
\end{aligned}
$$

The standard Lindblad equation for this model has been given in Ref. [52] (see also Refs. [53,54] for more general Lindblad forms for this model and their solutions). For the special case of the chosen initial state, the Lindblad equation becomes

$$
\begin{aligned}
\dot{\varrho}_{\mathrm{S}}(\tau)= & -i\left[\left(\omega_{0}+\delta\right) \hat{a}^{\dagger} \hat{a}, \varrho_{\mathrm{S}}(\tau)\right] \\
& +\gamma\left(2 \hat{a} \varrho_{\mathrm{S}}(\tau) \hat{a}^{\dagger}-\hat{a}^{\dagger} \hat{a} \varrho_{\mathrm{S}}(\tau)-\varrho_{\mathrm{S}}(\tau) \hat{a}^{\dagger} \hat{a}\right),
\end{aligned}
$$

where $\delta=\mathbb{P}\left[\int_{0}^{\infty} d \omega J(\omega)\left(\omega_{0}-\omega\right)^{-1}\right], \gamma=\pi J\left(\omega_{0}\right), J(\omega)=$ $\sum_{k=1}^{M} g_{k}^{2} \delta\left(\omega-\omega_{k}\right)$ (the spectral density function), and $\mathbb{P}$ denotes the Cauchy principal value.

To compare the MLL equation with the standard Lindblad equation, the ULL2 equation, and the exact

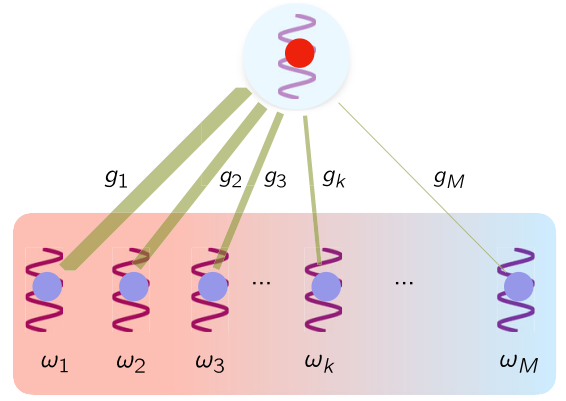

FIG. 3. Schematic model of a damped harmonic oscillator. See the text for details. 

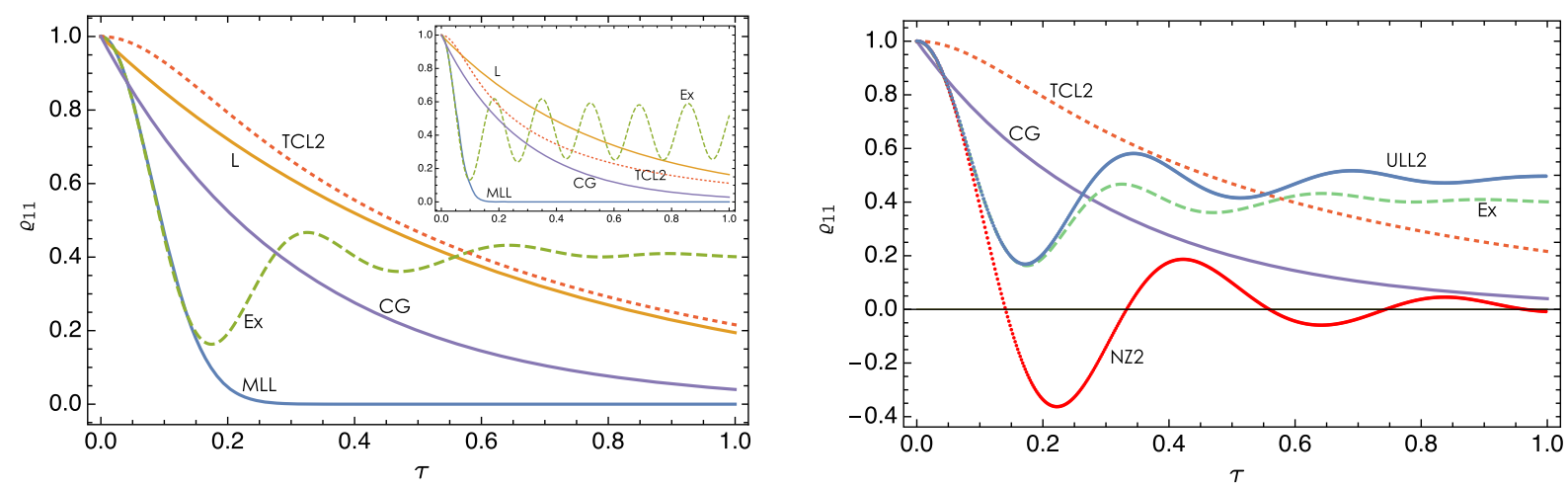

FIG. 4. Damped harmonic oscillator. Left panel: Population of the first excited state of the system harmonic oscillator vs time for $\omega_{0}=1$ and $\omega_{c}=5$ (all in dimensionless units) when the system is initially in the state $|\psi(0)\rangle_{S}=|1\rangle_{S}$ and the bath has infinite oscillators $(M \rightarrow \infty)$ at zero temperature. For the numerical solution of the exact dynamics, we have assumed $M=255, g_{k}=\sqrt{J\left(\omega_{k}\right)}$, and $\omega_{k}=0.1 k$, where $1 \leq k \leq M$. The labels CG, Ex, L, MLL, and TCL2 denote, respectively, data from the CG, exact, Lindblad, MLL, and TCL2 solutions. The inset shows an identical case, except that $\omega_{c}=10$. Right panel: The same as the left panel, but only for the ULL2, NZ2, CG, TCL2, and exact solutions.

dynamics, we choose an Ohmic spectral density as $J(\omega)=$ $(\omega / \pi) e^{-\omega / \omega_{c}}$ (similar to Ref. [52]). For numerical simulation of this model, we take $\omega_{k}=0.1 k$ and $M=255$. In order for the MLL equation and the simulations to be comparable with the standard Lindblad equation, we choose the coupling strength as $g_{k}=\sqrt{J\left(\omega_{k}\right)}$. It is shown in Fig. 4 (left panel) that the short-time dynamics is well captured by the MLL equation, for all chosen cutoff values, whereas the Lindblad equation fails to capture this and the TCL2 equation seems to capture only the initial moments of the dynamics. When $\omega_{c}=10$, the exact dynamics shows nondecaying oscillations, which may be due to nonMarkovianity. It is interesting to note that even in this non-Markovian case, the MLL equation-which is Markovian-can capture the short-time dynamics well. Considering the dynamics at long times, we observe in Fig. 4 (right panel) that the ULL2 equation can capture the exact dynamics with good accuracy, while the NZ2 equation [3] gives unphysical results. It is interesting that, although the complete ULL and the NZ dynamical equations are exact, their second-order approximations, i.e., ULL2 and NZ2, estimate the dynamics differently. This result is due to the different underlying approaches in applying the second-order approximation.

In addition, by calculating the population of the first excited state using the suggested asymptotic state $Q^{\star}$ [39], we obtain that $Q_{11}^{\star}=0.3963$ equals the asymptotic value in Fig. 4.

\section{SUMMARY AND CONCLUSIONS}

We have introduced the correlation picture as a new dynamical picture. By using the Schrödinger equation of the total system, using a correlating transformation, and tracing over the environment degrees of freedom, we have found the dynamical equation of the subsystem without invoking any approximations. We have shown that this exact dynamical equation is in the Lindblad form, even if the system is initially correlated or is in the strong-coupling regime. Hence, the Lindblad form for the dynamical equation is general, and the obtained master equation is a ULL equation. We have provided a way to derive a MLL from the ULL equation. In particular, we have shown that Markovianity can emerge if we apply a weak-correlation approximation, and the MLL equation becomes exact at instants with vanishing correlations. We have demonstrated that, not only at the initial time (if the system and the bath are prepared in a product state) but also in the asymptotic time, this weak-correlation approximation can be valid under certain conditions.

The correlation picture has also enabled us to formulate a systematic weak-correlation perturbative expansion, from which we have introduced approximate second- and higher-order tractable master equations. The MLL methodology plays an important role in this construction of the master equations, which can feature non-Markovian effects. We have shown that existing and widely used weak-coupling-based equations emerge as special cases of our perturbative constructions, and thus our weak-correlation master equations are expected to outperform or perform as accurately as corresponding weak-coupling solutions. In particular, we have illustrated through three examples our results for the existence of the ULL equation and the validity of the MLL equation around weakcorrelation points for initial and asymptotic times, and we have compared the MLL and ULL2 equations as approximate solutions with other Markovian and nonMarkovian equations. We have shown that, in these examples, our equations describe the dynamics more accurately. We expect that introducing the correlation picture can pave the way for developing new techniques for controlling and harnessing system-environment 
correlations. We also anticipate a wide range of applications of our theory from quantum thermodynamics to quantum computation. In particular, our approach may help one to understand whether and how quantum systems thermalize, and it may shed light on the role of correlations in quantum algorithms and the robustness of quantum error correction against correlated noise mechanisms.

\section{ACKNOWLEDGMENTS}

Discussions with J. Anders, E. Aurell, H.-P. Breuer, L. A. Correa, S. F. Huelga, A. Isar, S. J. Kazemi, S. Maniscalco, J. Piilo, Á. Rivas, R. Sampaio, J. Tuorila, and S. Vinjanampathy are acknowledged. This work was supported by the Academy of Finland's Center of Excellence program QTF Project 312057 and Sharif University of Technology's Office of Vice President for Research and Technology.

[1] F. Verstraete, M. M. Wolf, and J. I. Cirac, Quantum Computation and Quantum-State Engineering Driven by Dissipation, Nat. Phys. 5, 633 (2009).

[2] J. L. Herek, W. Wohlleben, R. J. Cogdell, D. Zeidler, and M. Motzkus, Quantum Control of Energy Flow in Light Harvesting, Nature (London) 417, 533 (2002).

[3] H.-P. Breuer and F. Petruccione, The Theory of Open Quantum Systems (Oxford University Press, New York, 2002).

[4] Á. Rivas and S.F. Huelga, Open Quantum Systems (Springer, Berlin, 2012).

[5] S. Nakajima, On Quantum Theory of Transport Phenomena: Steady Diffusion, Prog. Theor. Phys. 20, 948 (1958).

[6] R. Zwanzig, Ensemble Method in the Theory of Irreversibility, J. Chem. Phys. 33, 1338 (1960).

[7] I. de Vega and D. Alonso, Dynamics of Non-Markovian Open Quantum Systems, Rev. Mod. Phys. 89, 015001 (2017).

[8] H.-P. Breuer, J. Gemmer, and M. Michel, Non-Markovian Quantum Dynamics: Correlated Projection Superoperators and Hilbert Space Averaging, Phys. Rev. E 73, 016139 (2006).

[9] P. Haikka and S. Maniscalco, Non-Markovian Dynamics of a Damped Driven Two-State System, Phys. Rev. A 81, 052103 (2010).

[10] H.-P. Breuer, B. Kappler, and F. Petruccione, Stochastic Wave-Function Method for Non-Markovian Quantum Master Equations, Phys. Rev. A 59, 1633 (1999).

[11] J. Iles-Smith, A. G. Dijkstra, N. Lambert, and A. Nazir, Energy Transfer in Structured and Unstructured Environments: Master Equations beyond the Born-Markov Approximations, J. Chem. Phys. 144, 044110 (2016).

[12] F. A. Pollock and K. Modi, Tomographically Reconstructed Master Equations for Any Open Quantum Dynamics, Quantum 2, 76 (2018).

[13] G. A. Paz-Silva, M. J. W. Hall, and H. M. Wiseman, Dynamics of Initially Correlated Open Quantum Systems: Theory and Applications, Phys. Rev. A 100, 042120 (2019).
[14] J. Tuorila, J. Stockburger, T. Ala-Nissila, J. Ankerhold, and M. Möttönen, System-Environment Correlations in Qubit Initialization and Control, Phys. Rev. Research 1, 013004 (2019).

[15] M. J. W. Hall, J. D. Cresser, L. Li, and E. Andersson, Canonical Form of Master Equations and Characterization of Non-Markovianity, Phys. Rev. A 89, 042120 (2014).

[16] G. Lindblad, On the Generators of Quantum Dynamical Semigroups, Commun. Math. Phys. 48, 119 (1976).

[17] V. Gorini, A. Kossakowsi, and E. C. G. Sudarshan, Completely Positive Dynamical Semigroups of N-Level Systems, J. Math. Phys. (N.Y.) 17, 821 (1976).

[18] D. A. Lidar, Z. Bihary, and K. B. Whaley, From Completely Positive Maps to the Quantum Markovian Semigroup Master Equation, Chem. Phys. 268, 35 (2001).

[19] D. Chruściński and A. Kossakowski, Non-Markovian Quantum Dynamics: Local versus Nonlocal, Phys. Rev. Lett. 104, 070406 (2010).

[20] S. Weinberg, What Happens in a Measurement?, Phys. Rev. A 93, 032124 (2016).

[21] A. Bassi, D. Dürr, and G. Hinrichs, Uniqueness of the Equation for Quantum State Sector Collapse, Phys. Rev. Lett. 111, 210401 (2013).

[22] H. M. Wiseman and L. Diósi, Complete Parameterization, and Invariance, of Diffusive Quantum Trajectories for Markovian Open Systems, Chem. Phys. 268, 91 (2001).

[23] D. Braun, F. Haake, and W. T. Strunz, Universality of Decoherence, Phys. Rev. Lett. 86, 2913 (2001).

[24] M. Beau, J. Kiukas, I. L. Egusquiza, and A. del Campo, Nonexponential Quantum Decay under Environmental Decoherence, Phys. Rev. Lett. 119, 130401 (2017).

[25] Á. Rivas, Refined Weak-Coupling Limit: Coherence, Entanglement, and Non-Markovianity, Phys. Rev. A 95, 042104 (2017).

[26] It has been shown in R. Sampaio, J. Anders, T. G. Philbin, and T. Ala-Nissila, Contributions to Single-Shot Energy Exchanges in Open Quantum Systems, Phys. Rev. E 99, 062131 (2019), that the concept of a conditional wave function can be used to characterize the energy associated to correlation between two coupled quantum systems but with the dynamics complicated by a nonlinear Schrödinger equation.

[27] S. Alipour, S. Tuohino, A. T. Rezakhani, and T. Ala-Nissila, Unreliability of Mutual Information as a Measure in Total Variations, Phys. Rev. A 101, 042311 (2020).

[28] M. A. Nielsen and I. L. Chuang, Quantum Computation and Quantum Information (Cambridge University Press, Cambridge, England, 2000).

[29] L. Amico, R. Fazio, A. Osterloh, and V. Vedral, Entanglement in Many-Body Systems, Rev. Mod. Phys. 80, 517 (2008).

[30] K. Modi, A. Brodutch, H. Cable, T. Paterek, and V. Vedral, The Classical-Quantum Boundary for Correlations: Discord and Related Measures, Rev. Mod. Phys. 84, 1655 (2012).

[31] M. M. Wolf, Quantum Channels \& Operations-Guided Tour (Lecture Notes, 2012).

[32] G. M. D’Ariano, R. Demkowicz-Dobrzański, P. Perinotti, and M. F. Sacchi, Erasable and Unerasable Correlations, Phys. Rev. Lett. 99, 070501 (2007).

[33] D. R. Terno, Nonlinear Operations in Quantum-Information Theory, Phys. Rev. A 59, 3320 (1999). 
[34] J.-L. Brylinski and R. Brylinski, in Mathematics of Quantum Computation, edited by R. K. Brylinski and G. Chen (Chapman \& Hall/CRC, Boca Raton, 2002), pp. 101-116.

[35] We also request that $\operatorname{Tr}_{\mathrm{S}}\left[H_{\chi}, \varrho_{\mathrm{S}} \otimes \varrho_{\mathrm{B}}\right]=\operatorname{Tr}_{\mathrm{B}}\left[H_{\chi}, \varrho_{\mathrm{S}} \otimes\right.$ $\left.\varrho_{\mathrm{B}}\right]=0$ to ensure $\operatorname{Tr}_{\mathrm{S}}[\chi]=\operatorname{Tr}_{\mathrm{B}}[\chi]=0$.

[36] R. Wu, A. Pechen, C. Brif, and H. Rabitz, Controllability of Open Quantum Systems with Kraus-Map Dynamics, J. Phys. A 40, 5681 (2007).

[37] M. Mohseni and A. T. Rezakhani, Equation of Motion for the Process Matrix: Hamiltonian Identification and Dynamical Control of Open Quantum Systems, Phys. Rev. A 80, 010101(R) (2009).

[38] D.S. Djordjević, Explicit Solution of the Operator Equation $A^{*} X+X^{*} A=B$, J. Comput. Appl. Math. 200, 701 (2007).

[39] See Supplemental Material at http://link.aps.org/ supplemental/10.1103/PhysRevX.10.041024 for details and proofs.

[40] M. R. Hush, I. Lesanovsky, and J. P. Garrahan, Generic Map from Non-Lindblad to Lindblad Master Equations, Phys. Rev. A 91, 032113 (2015).

[41] C. C. Gerry and P. L. Knight, Introductory Quantum Optics (Cambridge University Press, Cambridge, England, 2005).

[42] P. Gaspard and M. Nagaoka, Slippage of Initial Conditions for the Redfield Master Equation, J. Chem. Phys. 111, 5668 (1999).

[43] R. S. Whitney, Staying Positive: Going beyond Lindblad with Perturbative Master Equations, J. Phys. A 41, 175304 (2008).

[44] A. S. L. Malabarba, L. P. García-Pintos, N. Linden, T. C. Farrelly, and A.J. Short, Quantum Systems Equilibrate
Rapidly for Most Observables, Phys. Rev. E 90, 012121 (2014).

[45] A. J. Short and T. C. Farrelly, Quantum Equilibration in Finite Time, New J. Phys. 14, 013063 (2012).

[46] S. Goldstein, T. Hara, and H. Tasaki, Extremely Quick Thermalization in a Macroscopic Quantum System for a Typical Nonequilibrium Subspace, New J. Phys. 17, 045002 (2015).

[47] L. P. García-Pintos, N. Linden, A. S. L. Malabarba, A. J. Short, and A. Winter, Equilibration Time Scales of Physically Relevant Observables, Phys. Rev. X 7, 031027 (2017).

[48] G. Schaller, Open Quantum Systems Far from Equilibrium (Springer International, Cham, Switzerland, 2014).

[49] G. Schaller and T. Brandes, Preservation of Positivity by Dynamical Coarse Graining, Phys. Rev. A 78, 022106 (2008).

[50] G. Schaller, P. Zedler, and T. Brandes, Systematic Perturbation Theory for Dynamical Coarse-Graining, Phys. Rev. A 79, 032110 (2009).

[51] C. Majenz, T. Albash, H.-P. Breuer, and D. A. Lidar, Coarse Graining Can Beat the Rotating-Wave Approximation in Quantum Markovian Master Equations, Phys. Rev. A 88, 012103 (2013).

[52] Á. Rivas, A. D. K. Plato, S. F. Huelga, and M. B. Plenio, Markovian Master Equations: A Critical Study, New J. Phys. 12, 113032 (2010).

[53] A. Isar, A. Sandulescu, and W. Scheid, Density Matrix for the Damped Harmonic Oscillator within the Lindblad Theory, J. Math. Phys. (N.Y.) 34, 3887 (1993).

[54] R. Karrlein and H. Grabert, Exact Time Evolution and Master Equations for the Damped Harmonic Oscillator, Phys. Rev. E 55, 153 (1997). 